\title{
Path-integral virial estimator based on the scaling of fluctuation coordinates: Application to quantum clusters with fourth-order propagators
}

\author{
$\operatorname{AUTHOR}(\mathrm{S})$ : \\ Yamamoto, TM
}

\section{CITATION:}

Yamamoto, TM. Path-integral virial estimator based on the scaling of fluctuation coordinates: Application to quantum clusters with fourth-order propagators. JOURNAL OF CHEMICAL PHYSICS 2005, 123(10): 104101.

ISSUE DATE:

2005-09-08

URL:

http://hdl.handle.net/2433/50584

\section{RIGHT:}

Copyright 2005 American Institute of Physics. This article may be downloaded for personal use only. Any other use requires prior permission of the author and the American Institute of Physics. 


\title{
Path-integral virial estimator based on the scaling of fluctuation coordinates: Application to quantum clusters with fourth-order propagators
}

\author{
Takeshi M. Yamamoto ${ }^{\text {a) }}$ \\ Department of Chemistry, Graduate School of Science, Kyoto University, Kyoto 606-8502, Japan
}

(Received 13 May 2005; accepted 13 July 2005; published online 9 September 2005)

\begin{abstract}
We first show that a simple scaling of fluctuation coordinates defined in terms of a given reference point gives the conventional virial estimator in discretized path integral, where different choices of the reference point lead to different forms of the estimator (e.g., centroid virial). The merit of this procedure is that it allows a finite-difference evaluation of the virial estimator with respect to temperature, which totally avoids the need of higher-order potential derivatives. We apply this procedure to energy and heat-capacity calculations of the $\left(\mathrm{H}_{2}\right)_{22}$ and $\mathrm{Ne}_{13}$ clusters at low temperature using the fourth-order Takahashi-Imada [J. Phys. Soc. Jpn. 53, 3765 (1984)] and Suzuki [Phys. Lett. A 201, 425 (1995)] propagators. This type of calculation requires up to third-order potential derivatives if analytical virial estimators are used, but in practice only first-order derivatives suffice by virtue of the finite-difference scheme above. From the application to quantum clusters, we find that the fourth-order propagators do improve upon the primitive approximation, and that the choice of the reference point plays a vital role in reducing the variance of the virial estimator. (C) 2005 American Institute of Physics. [DOI: 10.1063/1.2013257]
\end{abstract}

\section{INTRODUCTION}

Imaginary-time path integral provides a robust way for studying quantum statistical mechanics of many-particle systems. ${ }^{1,2}$ In the framework of discretized path integral, this method maps a quantum system into multiple copies of virtual classical systems (called "beads") connected via harmonic springs. This isomorphism allows one to calculate structural and thermodynamic properties using conventional Monte Carlo or molecular-dynamics methods. In practice, however, such calculation often becomes much more demanding than the classical counterpart, and thus a number of efficient techniques have been developed, e.g., collective sampling of multiple beads, ${ }^{2-5}$ statistical estimators with low variance, ${ }^{6-16}$ and accurate approximations to the exact shorttime propagator (or high-temperature density matrix). ${ }^{3,17-35}$

Our interest in this paper is in the latter two issues, namely, the use of better statistical estimators and approximate propagators. Regarding the estimator, the most conventional path-integral estimators for internal energy are thermodynamic $^{6}$ and virial ${ }^{7}$ estimators. The former is obtained via direct temperature differentiation of the partition function, while the latter is obtained by eliminating illbehaved terms in the former through integration by parts. The virial estimator has an advantage that its variance is only weakly dependent on the number of beads, $P$, in contrast with the thermodynamic estimator whose variance grows linearly with $P$. We emphasize, however, that this reduction in the variance is achieved at the expense of using first-order potential derivatives that are absent in the thermodynamic estimator. Although the first-order derivatives are often not a major computational problem, things become worse when

${ }^{a)}$ Electronic mail: yamamoto@kuchem.kyoto-u.ac.jp one constructs a similar double virial estimator for heat capacity because it requires second-order potential derivatives. Despite this difficulty, the double virial estimator was used in a heat-capacity calculation of water because other estimators exhibited too large statistical errors and could not be converged within simulation time. ${ }^{16}$ To remedy this problem, Glaesemann and Fried ${ }^{12,13}$ proposed a free-particle projection technique to reduce the variance of the thermodynamic estimator without using potential derivatives, and applied it to $\mathrm{Ar}_{6}$ clusters with considerable success at higher temperature. Predescu et al. ${ }^{15}$ adopted a different strategy in their random series path integral (generalized form of the Fourier path integral), where they first scaled the amplitude of the Brownian bridge and then differentiated the scaled partition function via finite-difference in order to obtain a virial-like estimator having no potential derivatives. With this method they calculated the quantum heat capacity of the Ne cluster at 4-14 K with unprecedented accuracy. ${ }^{15}$

Another issue that impacts the efficiency of path integral is the accuracy of approximate propagators. There exist a number of such approximations that aim at faster convergence to the $P \rightarrow \infty$ limit than the standard primitive approximation. In particular, the pair-product approximation ${ }^{2,3}$ and the higher-order composite factorizations ${ }^{17-19,23}$ have proven to be successful in condensed-phase applications (see Ref. 27 for their useful comparisons). The pair-product approximation replaces the exact high-temperature density matrix by the product of effective pairwise ones, and it has been shown to drastically reduce the number of beads for monoatomic fluids. ${ }^{34,36}$ The fast convergence of this approximation was also exploited in semiclassical dynamical calculation of normal and superfluid heliums. ${ }^{37}$ While powerful for monoatomic fluids, the pair-product approximation becomes cumbersome when applied to molecular fluids because of the 
increased complexity of pair action. In this regard the higherorder propagators are appealing in that the molecular fluids can be treated straightforwardly. In fact, however, the application of such propagators to molecular fluids is rather scant compared to the primitive approximation. One reason may be that the higher-order propagators involve the first-order potential derivatives, and the corresponding virial estimator for energy and heat capacity requires second- and third-order potential derivatives, respectively, resulting in a significant computational overhead. (Incidentally, Jang et al. ${ }^{23}$ showed that for the Suzuki propagator the required order of potential derivatives can be reduced by using the virial theorem in operator form.)

In this paper we present a method for evaluating the virial and double virial estimators in discretized path integral without using higher-order potential derivatives. This method is based on the coordinate scaling idea of Janke and Sauer ${ }^{10}$ and the finite-difference method of Predescu et al. ${ }^{15}$ Specifically, we first show that a simple scaling of fluctuation coordinates defined in terms of a given reference point gives the conventional virial estimator, where different choices of the reference point lead to different forms of the estimator (e.g., centroid virial). This procedure reverts to the original coordinate scaling by Janke et al. when the reference point is set to the coordinate origin. We then take the temperature derivative of the scaled partition function by finite difference in order to avoid potential derivatives. We illustrate the above procedure by calculating the energy and heat capacity of the $\left(\mathrm{H}_{2}\right)_{22}$ and $\mathrm{Ne}_{13}$ clusters at low temperature using the fourthorder composite propagators. This calculation requires up to third-order potential derivatives if analytical virial estimators are used, but in practice only up to first-order derivatives suffice by virtue of the finite-difference scheme above. From the results of the application, we find that the fourth-order propagators do improve upon the primitive approximation, and that the choice of the reference point has a crucial role in reducing the variance of the virial estimator.

The remainder of this paper is as follows: In Sec. II we describe the coordinate scaling and finite-difference procedures mentioned above. In Sec. III we apply the present method to the $\left(\mathrm{H}_{2}\right)_{22}$ cluster at $6 \mathrm{~K}$ and $\mathrm{Ne}_{13}$ cluster at 4-14 K and calculate their total energy, heat capacity, and distance distribution functions. Systematic comparisons are made among different types of propagators and estimators. In Sec. IV we conclude.

\section{PATH-INTEGRAL ESTIMATORS FOR ENERGY AND HEAT CAPACITY}

\section{A. Conventional estimators}

We first summarize the conventional thermodynamic and virial ${ }^{2,7,8}$ estimators for subsequent discussion. We suppose an $f$-dimensional system having the Hamiltonian $H$ $=T+V=\sum_{i=1}^{f} p_{i}^{2} / 2 m+V(\mathbf{x})$ with $\mathbf{x}=\left(x_{1}, \ldots, x_{f}\right)$. Using the primitive approximation to the canonical density operator,

$$
e^{-\epsilon \hat{H}}=e^{-\epsilon \hat{V} / 2} e^{-\epsilon \hat{T}} e^{-\epsilon \hat{V} / 2}+O\left(\epsilon^{3}\right),
$$

the partition function at inverse temperature $\beta=1 / k_{B} T$ can be written as

$$
\begin{aligned}
Z(\beta)= & \operatorname{tr}\left(e^{-\beta \hat{H}}\right)=\int d \mathbf{x}_{1} \cdots \int d \mathbf{x}_{P} \rho\left(\mathbf{x}_{1}, \ldots, \mathbf{x}_{P} ; \beta\right) \\
& +O\left(1 / P^{2}\right),
\end{aligned}
$$

with

$$
\begin{aligned}
\rho\left(\mathbf{x}_{1}, \ldots, \mathbf{x}_{P} ; \beta\right)= & \left(\frac{m P}{2 \pi \hbar^{2} \beta}\right)^{P f / 2} \exp \left\{-\frac{m P}{2 \hbar^{2} \beta} \sum_{s=1}^{P}\left(\mathbf{x}_{s}-\mathbf{x}_{s-1}\right)^{2}\right. \\
& \left.-\frac{\beta}{P} \sum_{s=1}^{P} V\left(\mathbf{x}_{s}\right)\right\},
\end{aligned}
$$

where $\mathbf{x}_{s}$ is the system coordinate in the sth time slice (or "beads") with $\mathbf{x}_{0}=\mathbf{x}_{P}$. The thermodynamic estimator is obtained by direct temperature differentiation of Eq. (2):

$$
E(\beta)=-\frac{1}{Z(\beta)} \frac{\partial Z(\beta)}{\partial \beta} \simeq\left\langle\varepsilon_{T}\right\rangle
$$

with

$$
\varepsilon_{T}=\frac{P f}{2 \beta}-\frac{m P}{2 \hbar^{2} \beta^{2}} \sum_{s=1}^{P}\left(\mathbf{x}_{s}-\mathbf{x}_{s-1}\right)^{2}+\frac{1}{P} \sum_{s=1}^{P} V\left(\mathbf{x}_{s}\right),
$$

where $\langle\cdots\rangle$ denotes an ensemble average over the sampling function $\rho\left(\mathbf{x}_{1}, \ldots, \mathbf{x}_{P} ; \beta\right)$. The drawback of this estimator is that its variance grows with $P$ due to cancellation of the first two terms in the right-hand side of Eq. (5). This difficulty can be avoided by using the relation,

$$
\begin{gathered}
\int d \mathbf{x}_{1} \cdots \int d \mathbf{x}_{P}\left[\sum_{s=1}^{P}\left(\mathbf{x}_{s}-\mathbf{x}^{*}\right) \cdot \frac{\partial}{\partial \mathbf{x}_{s}}\right] \rho\left(\mathbf{x}_{1}, \ldots, \mathbf{x}_{P} ; \beta\right) \\
=-(P-g) f \int d \mathbf{x}_{1} \cdots \int d \mathbf{x}_{P} \rho\left(\mathbf{x}_{1}, \ldots, \mathbf{x}_{P} ; \beta\right),
\end{gathered}
$$

which arises from integration by parts. In Eq. (6), $\mathbf{x}^{*}$ is a given "reference" point and $g$ is a constant that depends on the definition of $\mathbf{x}^{*}$. In this paper we consider three choices of $\mathbf{x}^{*}$, namely, $\mathbf{x}^{*}=0, \mathbf{x}_{P}$, and $\mathbf{x}_{c}$, where $\mathbf{x}_{c}$ is the centroid of the imaginary-time path given by

$$
\mathbf{x}_{c}=\frac{1}{P} \sum_{s=1}^{P} \mathbf{x}_{s} .
$$

With these choices the value of $g$ becomes ${ }^{38}$

$$
g= \begin{cases}0, & \mathbf{x}^{*}=0 \\ 1, & \mathbf{x}^{*}=\mathbf{x}_{P} \text { and } \mathbf{x}_{c} .\end{cases}
$$

Because the kinetic action in Eq. (3) is doubled by the "virial operator" in the square bracket in Eq. (6), ${ }^{39}$ the following path-integral virial theorem holds

$$
\begin{aligned}
& \left\langle\frac{m P}{2 \hbar^{2} \beta^{2}} \sum_{s=1}^{P}\left(\mathbf{x}_{s}-\mathbf{x}_{s-1}\right)^{2}+\frac{1}{2 P} \sum_{s=1}^{P}\left(\mathbf{x}_{s}-\mathbf{x}^{*}\right) \cdot \frac{\partial V\left(\mathbf{x}_{s}\right)}{\partial \mathbf{x}_{s}}\right\rangle \\
& \quad=\frac{(P-g) f}{2 \beta} .
\end{aligned}
$$

Eliminating the first two terms in Eq. (5) through the above relation, we have the following virial estimator for energy: 


$$
\varepsilon_{V}=\frac{f g}{2 \beta}+\frac{1}{P} \sum_{s=1}^{P}\left[\frac{1}{2}\left(\mathbf{x}_{s}-\mathbf{x}^{*}\right) \cdot \frac{\partial V\left(\mathbf{x}_{s}\right)}{\partial \mathbf{x}_{s}}+V\left(\mathbf{x}_{s}\right)\right] .
$$

For convenience we will refer to the above estimator with $\mathbf{x}^{*}=0, \mathbf{x}_{P}$, and $\mathbf{x}_{c}$ as the origin-, bead-, and centroid-reference virial estimators, respectively. We note that the originreference virial estimator gives an incorrect result for unbound systems ${ }^{2}$ (e.g., $\varepsilon_{V}$ vanishes for a free particle) although the bead- and centroid-reference virial estimators remain valid. The reason is that in the former the integral of $\rho\left(\mathbf{x}_{1}, \ldots, \mathbf{x}_{P} ; \beta\right)$ over the whole coordinate space is divergent, which invalidates Eq. (6), while in the latter the integration by parts can be performed in one less dimensions with some coordinate fixed (e.g., $\mathbf{x}_{P}$ in the bead-reference virial). Despite this deficiency, the origin-reference virial estimator can be applied to quantum clusters if the contribution of the center of mass is properly taken into account. ${ }^{11,14}$

Heat-capacity estimators can be obtained in a similar manner and are fully described in Ref. 13. The resulting double thermodynamic estimator contains no potential derivatives but its variance grows rapidly as $P^{2}$. The double virial estimator has a favorable variance weakly dependent on $P$ but it requires second-order potential derivatives, resulting in an increased computational cost ${ }^{16}$

\section{B. Virial estimator via coordinate scaling}

The virial estimator in Eq. (10) can also be obtained by a scaling of fluctuation coordinates as mentioned in the Introduction. This is achieved by first considering the partition function at a different temperature $\beta^{\prime}$,

$$
Z\left(\beta^{\prime}\right)=\int d \mathbf{x}_{1}^{\prime} \cdots \int d \mathbf{x}_{P}^{\prime} \rho\left(\mathbf{x}_{1}^{\prime}, \ldots, \mathbf{x}_{P}^{\prime} ; \beta^{\prime}\right),
$$

where $\rho$ is the density function in Eq. (3). To eliminate illbehaved terms in the thermodynamic estimator, we introduce a new set of variables $\left(\mathbf{x}_{1}, \ldots, \mathbf{x}_{P}\right)$ as

$$
\mathbf{x}_{s}^{\prime}=\mathbf{x}^{*}+\sqrt{\frac{\beta^{\prime}}{\beta}}\left(\mathbf{x}_{s}-\mathbf{x}^{*}\right)
$$

for $s=1, \ldots, P . \mathbf{x}^{*}$ in Eq. (12) is a reference point that has the same meaning as in the preceding section, i.e., $\mathbf{x}^{*}=0, \mathbf{x}_{P}$, or $\mathbf{x}_{c}$. The Jacobian of this transformation is

$$
d \mathbf{x}_{1}^{\prime} \cdots d \mathbf{x}_{P}^{\prime}=\left(\frac{\beta^{\prime}}{\beta}\right)^{(P-g) f / 2} d \mathbf{x}_{1} \cdots d \mathbf{x}_{P}
$$

where $g$ is given by Eq. (8). Since the transformation in Eq. (12) suggests

$$
\frac{1}{\beta^{\prime}} \sum_{s=1}^{P}\left(\mathbf{x}_{s}^{\prime}-\mathbf{x}_{s-1}^{\prime}\right)^{2}=\frac{1}{\beta} \sum_{s=1}^{P}\left(\mathbf{x}_{s}-\mathbf{x}_{s-1}\right)^{2}
$$

the partition function in Eq. (11) may be written as

$$
Z\left(\beta^{\prime}\right)=\int d \mathbf{x}_{1} \cdots \int d \mathbf{x}_{P} \rho\left(\mathbf{x}_{1}, \ldots, \mathbf{x}_{P} ; \beta\right) R\left(\beta^{\prime}\right),
$$

with

$$
R\left(\beta^{\prime}\right)=\left(\frac{\beta}{\beta^{\prime}}\right)^{f g / 2} \exp \left\{-\frac{1}{P} \sum_{s=1}^{P}\left[\beta^{\prime} V\left(\mathbf{x}_{s}^{\prime}\right)-\beta V\left(\mathbf{x}_{s}\right)\right]\right\} .
$$

Using the above equation the internal energy is obtained as follows:

$$
E(\beta)=-\left.\left\langle\frac{\partial R\left(\beta^{\prime}\right)}{\partial \beta^{\prime}}\right\rangle\right|_{\beta^{\prime}=\beta}=\left\langle\varepsilon_{V}\right\rangle,
$$

with

$$
\varepsilon_{V}=\frac{f g}{2 \beta}+\left.\frac{1}{P} \sum_{s=1}^{P} \frac{\partial}{\partial \beta^{\prime}}\left[\beta^{\prime} V\left(\mathbf{x}_{s}^{\prime}\left(\beta^{\prime}\right)\right)\right]\right|_{\beta^{\prime}=\beta},
$$

where we have explicitly denoted the $\beta^{\prime}$ dependence of $\mathbf{x}_{s}^{\prime}$. $\varepsilon_{V}$ in Eq. (18) can be shown identical to that in Eq. (10) by taking the $\beta^{\prime}$ derivative analytically. Instead, here we take the $\beta^{\prime}$ derivative via finite difference in order to avoid potential derivatives, ${ }^{15,40}$

$$
\begin{aligned}
\varepsilon_{V} \simeq & \frac{f g}{2 \beta}+\frac{1}{2 P \delta \beta} \sum_{s=1}^{P}\left[(\beta+\delta \beta) V\left(\mathbf{x}_{s}^{\prime}(\beta+\delta \beta)\right)\right. \\
& \left.-(\beta-\delta \beta) V\left(\mathbf{x}_{s}^{\prime}(\beta-\delta \beta)\right)\right] .
\end{aligned}
$$

Similarly, the constant volume heat capacity,

$$
C_{V}(\beta)=\frac{d E(T)}{d T}=k_{B} \beta^{2}\left\{\frac{1}{Z(\beta)} \frac{\partial^{2} Z(\beta)}{\partial \beta^{2}}-\left[\frac{1}{Z(\beta)} \frac{\partial Z(\beta)}{\partial \beta}\right]^{2}\right\},
$$

can be obtained using the following expression:

$$
C_{V}(\beta)=k_{B} \beta^{2}\left\{\left\langle\varepsilon_{V}^{2}\right\rangle-\left\langle\varepsilon_{V}\right\rangle^{2}-\left\langle\varepsilon_{V}^{\prime}\right\rangle\right\},
$$

with

$$
\varepsilon_{V}^{\prime}=-\frac{f g}{2 \beta^{2}}+\left.\frac{1}{P} \sum_{s=1}^{P} \frac{\partial^{2}}{\partial \beta^{\prime 2}}\left[\beta^{\prime} V\left(\mathbf{x}_{s}^{\prime}\left(\beta^{\prime}\right)\right)\right]\right|_{\beta^{\prime}=\beta},
$$

where we may use finite difference to evaluate the second derivative with respect to $\beta^{\prime}$. Although there are other schemes for performing finite difference, e.g.,

$$
E(\beta) \simeq-\left\langle\frac{R(\beta+\delta \beta)-R(\beta-\delta \beta)}{2 \delta \beta}\right\rangle,
$$

it has a narrower range of acceptable values of $\delta \beta$ than Eq. (19) due to the exponential behavior of $R\left(\beta^{\prime}\right)$. Therefore we will use only Eqs. (19) and (22) in the following sections.

We emphasize that the finite-difference scheme above is qualitatively different, e.g., from that performed for internal energy with respect to temperature,

$$
C_{V}(\beta) \simeq \frac{E(T+\delta T)-E(T-\delta T)}{2 \delta T} .
$$

where $E(T)$ contains statistical error and $\delta T$ must be taken sufficiently large so that $|E(T+\delta T)-E(T-\delta T)|$ is much larger than the statistical error in $E(T \pm \delta T)$. On the other hand, the finite difference in Eq. (19) is performed for a statistical error-free quantity, $\beta^{\prime} V\left[\mathbf{x}_{s}^{\prime}\left(\beta^{\prime}\right)\right]$, and thus $\delta \beta$ can 
be taken as small as machine precision allows. In practice, however, acceptable values of $\delta \beta$ may depend on the stiffness of the potential as well as thermodynamic conditions under study (e.g., particle density), so one needs to check the convergence by repeating a very short simulation with different values of $\delta \beta$. Our typical choice of $\delta \beta$ is $10^{-4} \beta$ (see Sec. III).

\section{Using fourth-order composite propagators}

An appealing feature of the finite-difference scheme in Sec. II B is that it does not require potential derivatives higher than those existing in the discretized action. This means that energy and heat capacity can be calculated with no potential derivatives when the primitive approximation is used, and only up to first-order derivatives are needed when the fourth-order composite propagators are used. The generalized Suzuki $^{17,18,23}$ and Takahashi-Imada ${ }^{19}$ approximations fall into the latter category. The Suzuki approximation factorizes the exact short-time propagator as

$$
e^{-2 \epsilon H}=e^{-\epsilon \tilde{V}_{e} / 3} e^{-\epsilon T} e^{-4 \epsilon \tilde{V}_{m} / 3} e^{-\epsilon T} e^{-\epsilon \tilde{V}_{e} / 3}+O\left(\epsilon^{5}\right),
$$

where $\tilde{V}_{m}$ and $\tilde{V}_{e}$ are effective potentials that involve firstorder potential derivatives (see Refs. 18 and 23 for details). With this factorization the approximate partition function becomes

$$
Z(\beta)=\int d \mathbf{x}_{1} \cdots \int d \mathbf{x}_{P} \rho^{(4)}\left(\mathbf{x}_{1}, \ldots, \mathbf{x}_{P} ; \beta\right)+O\left(1 / P^{4}\right),
$$

with

$$
\begin{aligned}
\rho^{(4)}\left(\mathbf{x}_{1}, \ldots, \mathbf{x}_{P} ; \beta\right)= & \left(\frac{m P}{2 \pi \hbar^{2} \beta}\right)^{P f / 2} \\
& \times \exp \left\{-\frac{m P}{2 \hbar^{2} \beta_{s=1}^{P}}\left(\mathbf{x}_{s}-\mathbf{x}_{s-1}\right)^{2}\right. \\
& \left.-\frac{\beta}{P} \sum_{s=1}^{P} w_{s} \tilde{V}_{s}\left(\mathbf{x}_{s} ; \beta\right)\right\},
\end{aligned}
$$

where $\widetilde{V}_{s}$ is a time-slice-dependent effective potential defined by

$$
\widetilde{V}_{s}(\mathbf{x} ; \beta)=V(\mathbf{x})+d_{s}(\beta / P)^{2} C(\mathbf{x}),
$$

with

$$
C(\mathbf{x})=[V,[T, V]]=\frac{\hbar^{2}}{m}\left|\frac{\partial V(\mathbf{x})}{\partial \mathbf{x}}\right|^{2}
$$

while $w_{s}$ and $d_{s}$ are a set of coefficients given by

$$
w_{s}= \begin{cases}2 / 3, & s=\text { even } \\ 4 / 3, & s=\text { odd },\end{cases}
$$

and

$$
d_{s}= \begin{cases}\alpha / 6, & s=\text { even } \\ (1-\alpha) / 12, & s=\text { odd }\end{cases}
$$

where $\alpha$ is an arbitrary parameter within $[0,1]$. The partition function for the Takahashi-Imada approximation ${ }^{19}$ can also be expressed in the form (27) with $w_{s}=1$ and $d_{s}=1 / 24$, although this approximation is not based on a genuine factorization such as Eq. (25). The fourth-order approximation in Eq. (26) differs from the primitive, second-order one only in that the bare potential is replaced by the slice-dependent effective potential in Eq. (28), and that the weight factors $\left\{w_{s}\right\}$ are introduced in a way similar to Simpson's quadrature rule. It is thus straightforward to apply the procedure in Sec. II B to obtain finite-difference virial estimators having no higherorder potential derivatives. Specifically, the statistical average is now taken over $\rho^{(4)}$ in Eq. (27), and we modify $R\left(\beta^{\prime}\right)$ in Eq. (16) as

$$
\begin{aligned}
R\left(\beta^{\prime}\right)= & \left(\frac{\beta}{\beta^{\prime}}\right)^{f g / 2} \exp \left\{-\frac{1}{P} \sum_{s=1}^{P} w_{s}\left[\beta^{\prime} \tilde{V}_{s}\left(\mathbf{x}_{s}^{\prime} ; \beta^{\prime}\right)\right.\right. \\
& \left.\left.-\beta \tilde{V}_{s}\left(\mathbf{x}_{s} ; \beta\right)\right]\right\},
\end{aligned}
$$

and the virial estimators in Eqs. (18) and (22) as follows:

$$
\begin{aligned}
& \varepsilon_{V}=\frac{f g}{2 \beta}+\left.\frac{1}{P} \sum_{s=1}^{P} w_{s} \frac{\partial}{\partial \beta^{\prime}}\left[\beta^{\prime} \tilde{V}_{s}\left(\mathbf{x}_{s}^{\prime} ; \beta^{\prime}\right)\right]\right|_{\beta^{\prime}=\beta}, \\
& \varepsilon_{V}^{\prime}=-\frac{f g}{2 \beta^{2}}+\left.\frac{1}{P} \sum_{s=1}^{P} w_{s} \frac{\partial^{2}}{\partial \beta^{\prime 2}}\left[\beta^{\prime} \tilde{V}_{s}\left(\mathbf{x}_{s}^{\prime} ; \beta^{\prime}\right)\right]\right|_{\beta^{\prime}=\beta} .
\end{aligned}
$$

\section{APPLICATION TO QUANTUM CLUSTERS}

\section{A. $\left(\mathrm{H}_{2}\right)_{22}$ cluster at $6 \mathrm{~K}$}

We illustrate the above procedure by first calculating the energy and heat capacity of the $\left(\mathrm{H}_{2}\right)_{22}$ cluster at $6 \mathrm{~K}$. The physical model is identical to that used in the previous studies. $^{36,41,42}$ Briefly, the system potential consists of Lennard-Jones (LJ) pair interactions with $\epsilon_{\mathrm{LJ}}=34.2 \mathrm{~K}$ and $\sigma_{\mathrm{LJ}}=2.96 \AA$, where the hydrogen molecules are treated as distinguishable spherical particles with their mass being 2 amu. Since a cluster in vacuum at any positive temperature is metastable with respect to evaporation, a confining potential of the form

$$
V_{c}=\epsilon_{\mathrm{LJ}} \sum_{i=1}^{N}\left(\frac{\left|\mathbf{r}_{i}-\mathbf{R}\right|}{R_{c}}\right)^{20}
$$

is added to the sum of the LJ potentials to prevent any molecules from permanently leaving the cluster. In Eq. (35), $\mathbf{r}_{i}$ is the position of particle $i, \mathbf{R}$ is the center of mass of the cluster, $N$ is the number of particles, and $R_{c}$ is the confining radius chosen as $4 \sigma_{\mathrm{LJ}}$.

Statistical sampling of imaginary-time paths was performed with Monte Carlo (MC) methods, where 1 cycle is defined such that each particle is moved once on average by the staging algorithm. ${ }^{3-5}$ The implementation is the same as 
TABLE I. Internal energy (K/molecule) of the $\left(\mathrm{H}_{2}\right)_{22}$ cluster at $6 \mathrm{~K}$ calculated using the centroid virial estimator based on the finite difference scheme in Sec. II B. PA, TIA, and SA denote the primitive, Takahashi-Imada, and Suzuki approximations, respectively. $\alpha$ is an arbitrary parameter within $[0,1]$ involved in the Suzuki approximation. The figure in parentheses is one standard deviation on the last digit.

\begin{tabular}{rccccc}
\hline \hline $\mathrm{P}$ & PA & TIA & SA $(\alpha=0)$ & SA $(\alpha=1 / 2)$ & SA $(\alpha=1)$ \\
\hline 20 & $-27.52(1)$ & $-21.80(1)$ & $-23.24(1)$ & $-20.94(1)$ & $-21.66(1)$ \\
40 & $-21.54(1)$ & $-18.80(1)$ & $-19.41(1)$ & $-18.34(1)$ & $-18.38(1)$ \\
60 & $-19.78(1)$ & $-18.13(1)$ & $-18.41(1)$ & $-17.86(1)$ & $-17.78(1)$ \\
80 & $-18.98(1)$ & $-17.90(1)$ & $-18.08(1)$ & $-17.72(1)$ & $-17.61(1)$ \\
100 & $-18.56(1)$ & $-17.79(1)$ & $-17.90(1)$ & $-17.66(1)$ & $-17.60(1)$ \\
120 & $-18.34(1)$ & $-17.76(1)$ & $-17.84(1)$ & $-17.68(1)$ & $-17.63(1)$ \\
160 & $-18.09(1)$ & $-17.73(1)$ & $-17.76(1)$ & $-17.68(1)$ & $-17.65(1)$ \\
\hline \hline
\end{tabular}

described in Ref. 5. The staging length $j$ (the number of beads that are collectively moved) was determined by adjusting the acceptance ratio to $50 \%$, which resulted in $j \approx P / 4$ regardless of the value of $P$. In addition to the staging move, we applied the whole-chain move ${ }^{2}$ every 2 cycles to accelerate the statistical convergence. A single run consisted of $5 \times 10^{5}$ cycles for equilibration followed by $4 \times 10^{6}$ cycles for data accumulation, which took several days for $P=160$ using a Pentium 4 3.8-GHz workstation.

Tables I and II list the energy and heat capacity obtained using the primitive, Takahashi-Imada, and Suzuki approximations. Figure 1 illustrates the systematic convergence of those values to the $P \rightarrow \infty$ limit. The centroid-reference virial estimator was used throughout based on the finite-difference scheme presented in Sec. II B. The relative statistical error was estimated to be on the order of $0.1 \%$ and $1 \%$ for energy and heat capacity, respectively, by using a blocking procedure with 2000 blocks each of 2000 cycles. The present calculation needed only up to first-order potential derivatives as mentioned in the Introduction. Acceptable values of the stepsize $\delta \beta$ ranged broadly from $10^{-3} \beta$ to $10^{-6} \beta$, where the smallest value was determined by round-off errors in the heat capacity. In this paper we set $\delta \beta$ to $10^{-4} \beta$, which practically gave the same result as when the analytical virial estimator was used.

Figure 1 shows that the fourth-order approximations improve remarkably upon the primitive approximation for both the energy and heat capacity. For example, to achieve a systematic error in the energy less than $0.25 \mathrm{~K} /$ molecule, ${ }^{36,41,42}$ the primitive approximation requires $P>200$ while the $\mathrm{Su}$ zuki approximation having $\alpha \gtrsim 0.5$ attains the same accuracy with $P=60$, thus reducing the necessary value of $P$ by a factor of $\sim 3$. This acceleration of systematic convergence is similar to that observed by Brualla et al. ${ }^{29}$ in the study of liquid ${ }^{4} \mathrm{He}$ at $5.1 \mathrm{~K}$ using the Takahashi-Imada approximation. Regarding the converged values of internal energy, the present result $(E=-17.68 \pm 0.01 \mathrm{~K} /$ molecule $)$ obtained using the Suzuki approximation with $P=160$ and $\alpha=0.5$ is in excellent agreement with the most accurate estimate $(E=-17.69 \pm 0.01 \mathrm{~K} /$ molecule $)$ obtained by Predescu et al. ${ }^{42}$ using the Wiener-Fourier reweighted path-integral method. Comparing different fourth-order propagators, we see that the Suzuki propagator with $\alpha=0.5$ and 1.0 converges somewhat faster than that with $\alpha=0.0$ or the Takahashi-Imada approximation.

Figure 2 compares statistical errors in the energy and heat capacity obtained with different estimators. The discretization was performed using the Suzuki approximation with $\alpha=0.5$. This figure shows that the thermodynamic estimator has a growing variance with $P$ while the virial estimators have a nearly constant variance. We should note, however, that the variance of the virial estimator strongly depends on the choice of the reference point $\mathbf{x}^{*}$ in Eq. (12). That is, the origin-reference virial estimator exhibits a significantly larger variance than the bead-or centroid-reference estimators, indicating that it is more advantageous to choose $\mathbf{x}^{*}$ in Eq. (12) as $\mathbf{x}_{P}$ or $\mathbf{x}_{c}$ than the coordinate origin.

Figure 2 also plots the statistical error of the modified centroid virial estimator that may be used in path-integral molecular dynamics. ${ }^{5,8}$ In the latter method the fourth-order composite propagators become expensive if $\rho^{(4)}$ in Eq. (27) is used directly as a sampling function, because the "forces" exerted on the beads require second-order potential derivatives. This problem can be avoided, for example, by excluding the force square terms in Eq. (29) from the sampling

TABLE II. Heat capacity (in unit of $k_{B}$ ) of the $\left(\mathrm{H}_{2}\right)_{22}$ cluster at $6 \mathrm{~K}$ calculated with the finite difference scheme in Sec. II B. Other details are the same as in Table I.

\begin{tabular}{rccccc}
\hline \hline $\mathrm{P}$ & $\mathrm{PA}$ & TIA & SA $(\alpha=0)$ & SA $(\alpha=1 / 2)$ & SA $(\alpha=1)$ \\
\hline 20 & $80.6(4)$ & $59.3(5)$ & $65.5(5)$ & $58.8(5)$ & $64.2(5)$ \\
40 & $55.5(4)$ & $44.5(5)$ & $47.4(4)$ & $42.5(5)$ & $44.4(5)$ \\
60 & $47.7(4)$ & $38.9(4)$ & $40.9(4)$ & $36.9(5)$ & $36.4(5)$ \\
80 & $42.5(4)$ & $37.7(4)$ & $38.5(4)$ & $35.8(4)$ & $35.1(5)$ \\
100 & $40.7(4)$ & $35.8(4)$ & $37.3(4)$ & $35.2(4)$ & $34.5(4)$ \\
120 & $39.3(4)$ & $35.7(4)$ & $35.6(4)$ & $34.9(4)$ & $34.8(4)$ \\
160 & $37.6(4)$ & $34.6(4)$ & $35.5(4)$ & $34.3(4)$ & $33.6(4)$ \\
\hline \hline
\end{tabular}



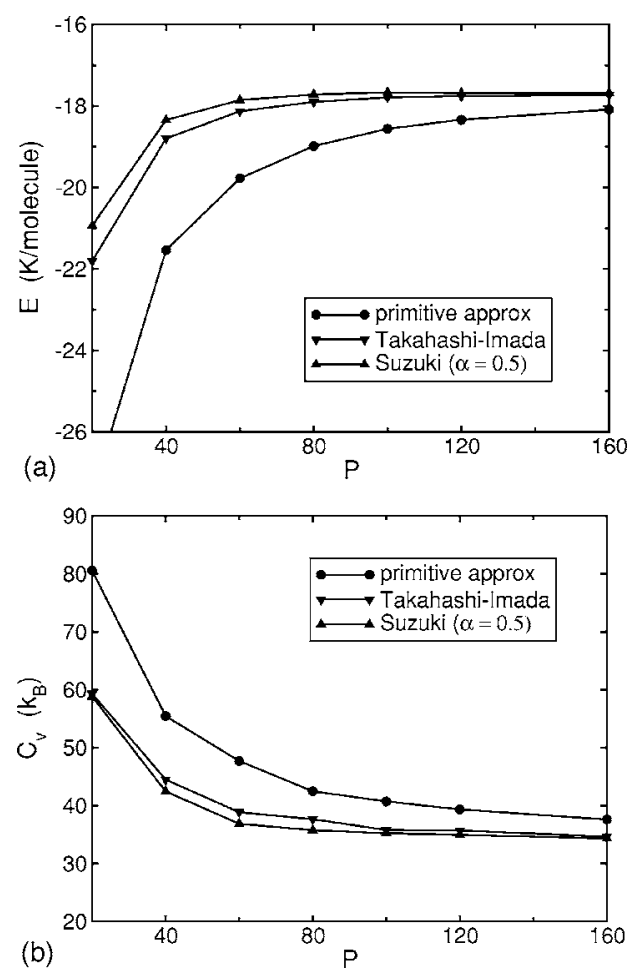

FIG. 1. Systematic convergence of (a) energy (K/molecule) and (b) heat capacity (in unit of $k_{B}$ ) of the $\left(\mathrm{H}_{2}\right)_{22}$ cluster at $6 \mathrm{~K}$ as a function of the Trotter number $P$.

function. ${ }^{23}$ The resulting modified expression for the energy is

$$
E(\beta) \simeq-\frac{\left\langle\Delta \rho \varepsilon_{V}\right\rangle_{\bmod }}{\langle\Delta \rho\rangle_{\bmod }}
$$

with

$$
\Delta \rho=\exp \left\{-\sum_{s=1}^{P} w_{s} d_{s}(\beta / P)^{3} C\left(\mathbf{x}_{s}\right)\right\},
$$

where various symbols are the same as in Sec. II C $\langle\cdots\rangle_{\bmod }$ in Eq. (36) denotes an ensemble average over the following sampling function:

$$
\begin{aligned}
\rho_{\text {mod }}\left(\mathbf{x}_{1}, \ldots, \mathbf{x}_{P}\right)= & \exp \left\{-\frac{m P}{2 \hbar^{2} \beta_{s=1}^{P}}\left(\mathbf{x}_{s}-\mathbf{x}_{s-1}\right)^{2}\right. \\
& \left.-\frac{\beta}{P} \sum_{s=1}^{P} w_{s} V\left(\mathbf{x}_{s}\right)\right\} .
\end{aligned}
$$

This method gives the true expected value of energy as $P$ is increased, but the statistical error becomes larger than the original scheme in Sec. II. ${ }^{23}$ Figure 2 shows that the variance obtained with this method is quite large for small values of $P$ but is reduced to a manageable size if $P$ is increased to $>40$. Thus, excluding force square terms from the sampling function seems a viable option if molecular-dynamics methods are used as a statistical sampler.

Figure 3 illustrates the classical and quantum results of the pair radial distribution function and the distance distribution function from the cluster center of mass (c.m.) defined by
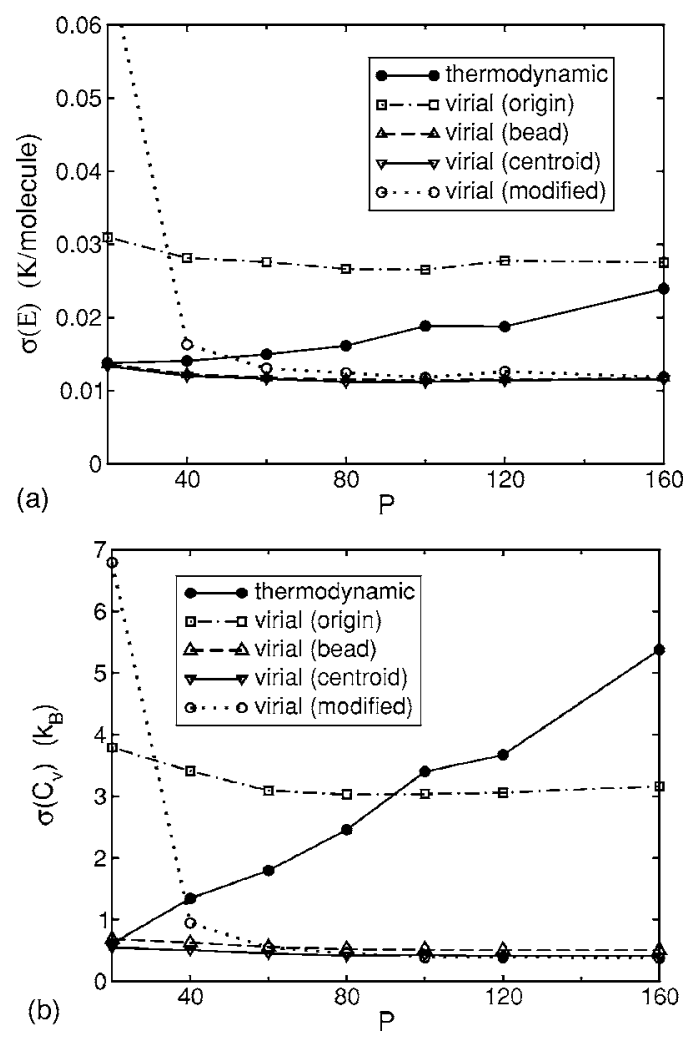

FIG. 2. Statistical error in (a) energy (K/molecule) and (b) heat capacity (in unit of $k_{B}$ ) of the $\left(\mathrm{H}_{2}\right)_{22}$ cluster at $6 \mathrm{~K}$ as a function of the Trotter number $P$. Five different estimators are compared: the thermodynamic estimator, the virial estimator with different choices of the reference point, and a modified virial estimator in Eq. (36). Errors in the heat capacity were estimated using the prescription given in Ref. 15.

$$
p_{\text {pair }}(r) \propto\left\langle\sum_{i<j}^{N} \sum_{s} \delta\left(r-r_{i j}^{(s)}\right)\right\rangle
$$

and

$$
p_{\text {c.m. }}(r) \propto\left\langle\sum_{i=1}^{N} \sum_{s} \delta\left(r-\Delta r_{i}^{(s)}\right)\right\rangle,
$$

respectively, where $r_{i j}=\left|\mathbf{r}_{i}-\mathbf{r}_{j}\right|$ and $\Delta r_{i}=\left|\mathbf{r}_{i}-\mathbf{R}\right|$. The sum over time slices is performed for all (only even) values of $s$ when the primitive (Suzuki) approximation is used. ${ }^{23}$ Here we do not consider the Takahashi-Imada approximation because it requires a nontrivial modification to the estimator. ${ }^{27}$ We see from Fig. 3 that the classical cluster has a rigid, solidlike structure at this temperature, ${ }^{43}$ while the quantum cluster has a liquidlike structure due to large zero-point energies and tunneling effects. This figure also shows that both the primitive and Suzuki approximations with $P=20$ already give a good approximation to the practically exact result obtained with $P=160$, indicating that structural properties converge much faster than the energy and heat capacity as a function of $P$.

\section{B. $\mathrm{Ne}_{13}$ cluster at $4-14 \mathrm{~K}$}

$\mathrm{Ne}_{13}$ is one of the smallest clusters that exhibit solidliquid-like (or melting) transition, and it has been studied extensively using a variety of theoretical methods. . $^{14,15,44-48}$ 

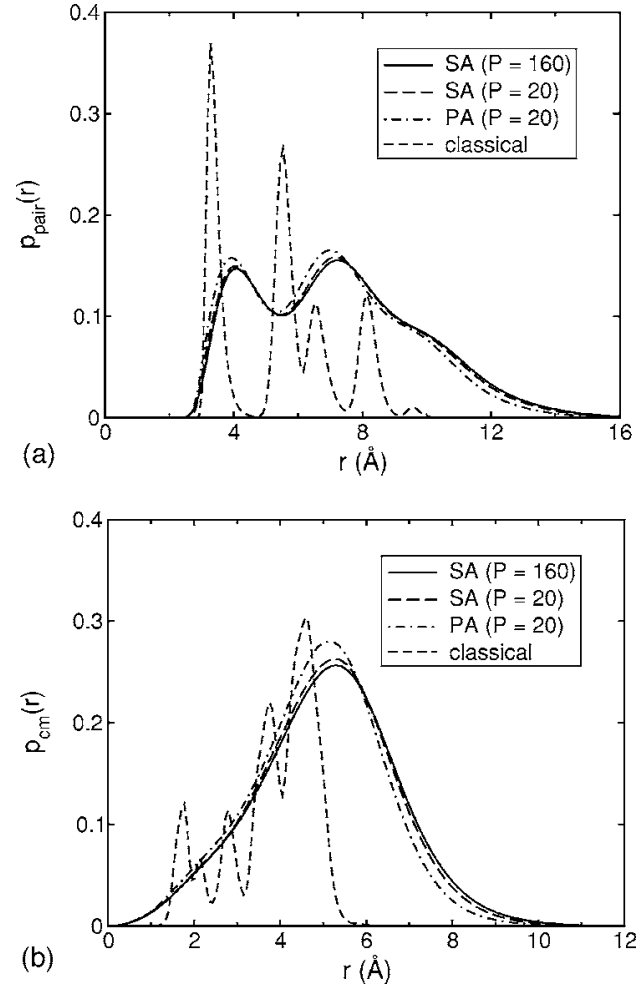

FIG. 3. (a) Pair radial distribution function and (b) distance distribution function from the center of mass of the $\left(\mathrm{H}_{2}\right)_{22}$ cluster at $6 \mathrm{~K}$. SA and PA denote the quantum results obtained with the Suzuki and primitive approximations, respectively. The classical result is plotted in short dashed line (with its height scaled by a factor of $1 / 2$ to fit in the panel).

The classical melting point is located at around $10 \mathrm{~K}$ and it is lowered by about $10 \%$ due to prominent quantum effects. The heat capacity is a useful quantity for characterizing such a cluster phase transition. Neirotti et al. ${ }^{14}$ calculated the heat capacity of $\mathrm{Ne}_{13}$ using a double virial estimator (in analytical form) designed for the Fourier path integral, but unfortunately their results exhibited a large statistical error (about $10 k_{B}$ ) in the low-temperature region. Predescu et al. ${ }^{15}$ calculated the same quantity using their virial-like estimator (in finite-difference form) in the framework of random series path integral, and as mentioned in the Introduction they obtained highly converged results with statistical errors less than $1 k_{B}$. Because the two calculations used the same number of Monte Carlo samples, this reduction in statistical error corresponds roughly to 100 times acceleration in convergence rate. Then a natural question that arises is what is the dominant factor that reduced the statistical error. We find, however, that this question is rather difficult to answer because there are quite a few technical differences in their calculations.

As such, to get some insights into the above question, we have recalculated the heat capacity of $\mathrm{Ne}_{13}$ using the discretized path integral. The computational details are basically the same as in the preceding section, and the relevant parameters were set as closely as possible to those in Refs. 14 and 15. Specifically, the Lennard-Jones parameters were set to $\epsilon_{\mathrm{LJ}}=35.6 \mathrm{~K}$ and $\sigma_{\mathrm{LJ}}=2.749 \AA$, and the mass of $\mathrm{Ne}$ was $20.0 \mathrm{amu}$. The confining radius $R_{c}$ in Eq. (35) was chosen as $2 \sigma_{\mathrm{LJ}}$. The number of Monte Carlo cycles was $4 \times 10^{6}$. We also performed the replica-exchange (or parallel tempering) Monte Carlo ${ }^{49-51}$ to avoid nonergodicity problem at low temperature. The number of replicas was set to 21 , and the replica temperatures were distributed over the interval $[4,14] \mathrm{K}$ with even spacing. The exchange move was attempted every Monte Carlo cycles. This setting ensured the acceptance ratio of exchange moves to be $>10 \%$.

Figure 4 plots the heat capacity thus obtained as a function of temperature. Four combinations of the approximate propagator and the heat-capacity estimator are examined, namely, (a) primitive approximation + double thermodynamic estimator, (b) primitive approximation+origin-reference double virial estimator, (c) primitive approximation+bead-reference double virial estimator, and (d) Suzuki approximation $(\alpha=0.5)+$ centroid-reference double virial estimator.

In cases (b), (c), and (d) the virial estimator was evaluated using the finite-difference scheme in Sec. II B, while in case (a) the heat capacity was calculated using the double thermodynamic estimator

$$
C_{V}(\beta)=k_{B} \beta^{2}\left\{\left\langle\varepsilon_{T}^{2}\right\rangle-\left\langle\varepsilon_{T}\right\rangle^{2}-\left\langle\frac{\partial \varepsilon_{T}}{\partial \beta}\right\rangle\right\},
$$

where $\varepsilon_{T}$ is given by Eq. (5). In all cases the number of time slices was set to $P=8-24$. Also shown in Fig. 4 is the highly accurate results obtained by Predescu et al. ${ }^{15}$ (circles) and the classical heat capacity (dotted line). This figure reveals that the origin-reference virial estimator has much larger statistical errors than the other three cases. Also interesting is the fact that the variance of the double thermodynamic estimator is rather small and close to the bead- and centroidreference double virial estimators. This tendency is qualitatively similar to that observed for the heat capacity of the hydrogen cluster in Fig. 2, where the origin-reference virial estimator has the largest statistical error in the small $P$ region. Regarding the systematic convergence to the $P \rightarrow \infty$ limit, Fig. 4(d) shows that the Suzuki propagator again provides a noticeable improvement over the primitive approximation, and that $P=20$ is sufficient to reach systematic convergence within $1 k_{B}$.

What is more important about the question discussed above is that cases (b) and (c) correspond qualitatively to the calculation by Neirotti et al. ${ }^{14}$ and Predescu et al.,${ }^{15}$ respectively. More precisely, the double virial estimator of Neirotti et al. may be regarded as origin reference because their estimator vanishes when the interaction potential is set to 0 (and thus the contribution of the center of mass was treated separately), while the finite-difference estimator of Predescu et al. may be viewed as bead reference because the relevant Brownian bridge is defined in terms of a set of "physical coordinates" (equivalent to a single bead). Thus, we think that the dominant factor that made a large difference in their calculations is the choice of the reference point in the virial estimator, rather than whether the estimator was evaluated analytically ${ }^{14}$ or numerically via finite difference ${ }^{15}$ if we consider the fact that cases (b), (c), and (d) above were treated using the finite-difference method in Sec. II B.

Finally, Fig. 5 illustrates the pair distribution functions at 4 and $10 \mathrm{~K}$, which are very similar to those presented in 

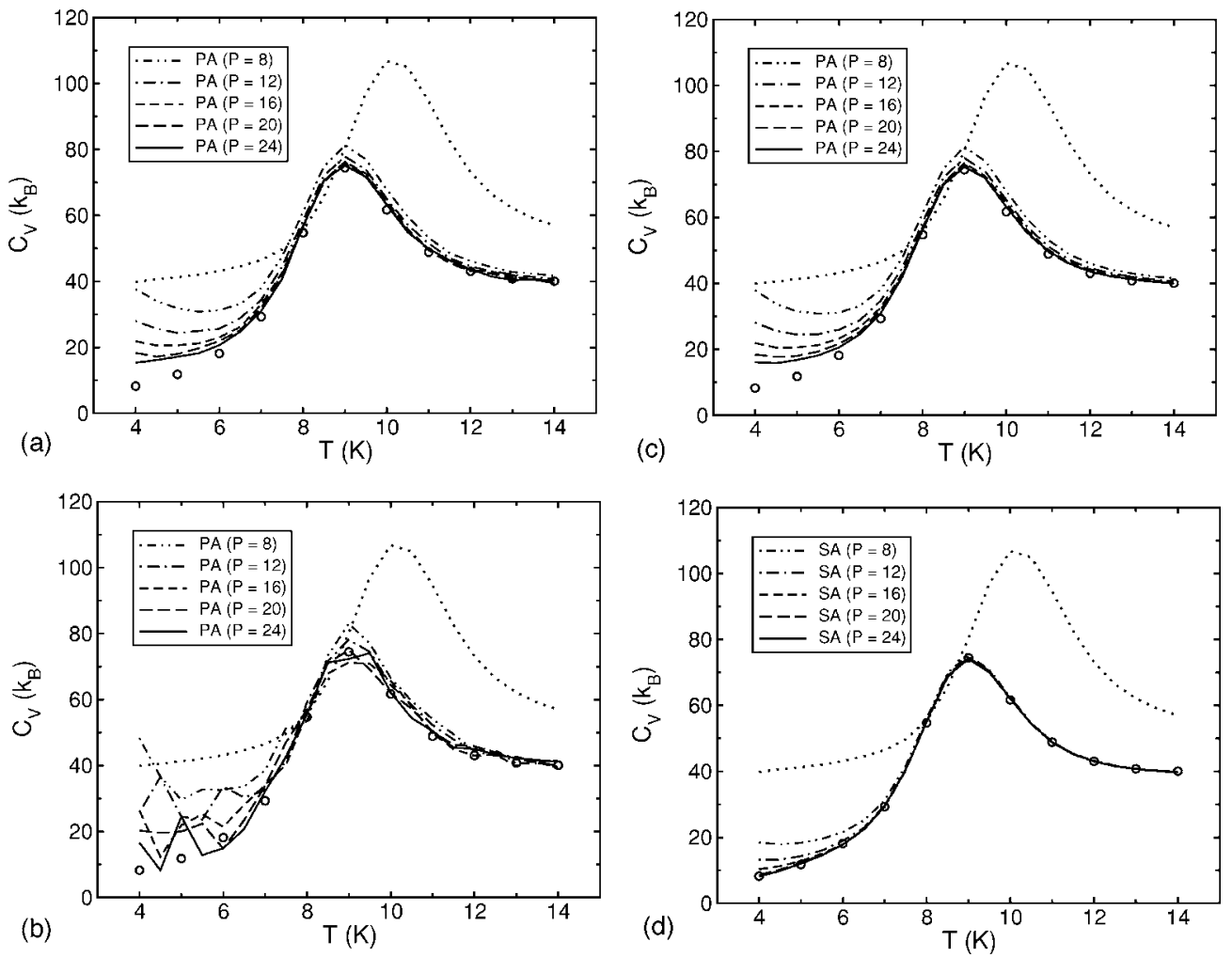

FIG. 4. Heat capacity of the $\mathrm{Ne}_{13}$ cluster as a function of temperature. SA and PA denote the quantum results obtained with the Suzuki and primitive approximations, respectively. Different estimators are used in each panel: (a) double thermodynamic estimator in Eq. (41); (b) origin-reference double virial estimator; (c) bead-reference double virial estimator; (d) centroid-reference double virial estimator. The statistical errors in panels (a), (c), and (d) are comparable with the width of the line while that in panel (b) is about $5 k_{B}$ in the low temperature region. The highly accurate results obtained by Predescu $e t$ al. (Ref. 15) are plotted by circles. The classical result is plotted in dotted line.

Refs. 46 and 47 . The cluster takes a solidlike structure at $4 \mathrm{~K}$ while it starts to form a liquidlike structure at $10 \mathrm{~K}$ (slightly above the melting temperature). Comparing the classical and quantum results, we see that the positions of the classical peaks are shifted outward and their widths broadened when quantum effects are made operative. The degree of broadening is much smaller than that observed for the hydrogen cluster in Fig. 3 due to the quasiclassical nature of $\mathrm{Ne}_{13}$. Figure 5 also shows that the primitive and Suzuki approximations with $P=24$ give almost indistinguishable results, verifying the fast convergence of structural properties with respect to $P$.

\section{CONCLUSIONS}

In this paper we have presented a coordinate scaling procedure for obtaining the conventional virial estimator and discussed its efficient evaluation using finite difference with respect to temperature. This procedure allowed us to apply the fourth-order propagators to quantum clusters using only the first-order potential derivatives. From the results of the application, we find that setting the reference point in the virial estimator to the coordinate origin (the path centroid) gives the largest (smallest) statistical errors. This result is also in qualitative agreement with previous studies on $\mathrm{Ar}$ clusters and liquid water. ${ }^{12,13,16}$ Thus, despite its extensive use in the literature, it is not recommended to use the originreference virial estimator in quantum clusters and condensed phase systems because of the large variance as well as un-
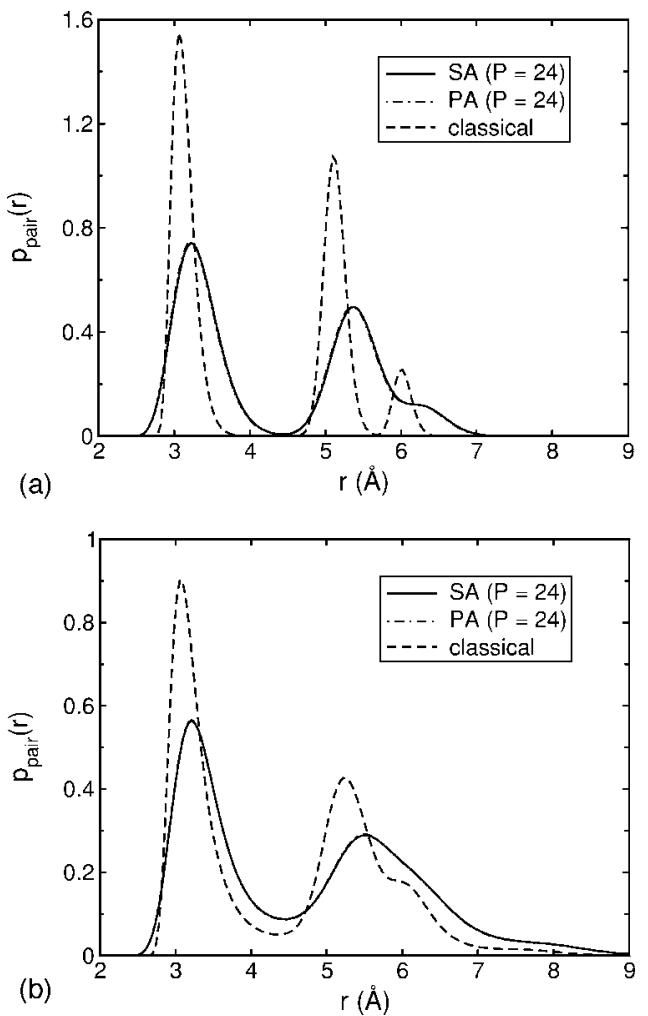

FIG. 5. Pair radial distribution functions of the $\mathrm{Ne}_{13}$ cluster at (a) $4 \mathrm{~K}$ and (b) $10 \mathrm{~K}$. SA and PA denote the quantum results obtained with the Suzuki and primitive approximations, respectively. The classical result is plotted in dashed line. 
necessary complication due to unbound degrees of freedom.

Finally, we mention some possible application of the present method. One example is a short-time approximation to the quantum correlation function $C_{A B}(t)$, e.g.,

$$
\begin{aligned}
C_{A B}(t) & =C_{A B}(0)+\frac{1}{2} \ddot{C}_{A B}(0) t^{2}+\cdots \\
& \simeq C_{A B}(0) \exp \left[\frac{1}{2} \frac{\ddot{C}_{A B}(0)}{C_{A B}(0)} t^{2}\right] .
\end{aligned}
$$

Taking the real-time derivatives of $C_{A B}(t)$ at $t=0$ along the imaginary-time axis results in a path-integral calculation similar to that of heat capacity, which implies a similar reduction in statistical errors via coordinate scaling. Such an idea is particularly relevant, e.g., to an approximate calculation of chemical reaction rates ${ }^{52-56}$ or vibrational relaxation rates. ${ }^{57}$ Another possible application is the path-integral ground state (or variational path-integral) methods, $2,58,59$ where several different estimators arise in natural analogy to finite temperature path integral.

${ }^{1}$ R. P. Feynman and A. R. Hibbs, Quantum Mechanics and Path Integrals (McGraw-Hill, New York, 1965).

${ }^{2}$ D. M. Ceperley, Rev. Mod. Phys. 67, 279 (1995).

${ }^{3}$ E. L. Pollock and D. M. Ceperley, Phys. Rev. B 30, 2555 (1984).

${ }^{4}$ M. Sprik, M. L. Klein, and D. Chandler, Phys. Rev. B 31, 4234 (1985).

${ }^{5}$ M. E. Tuckerman, B. J. Berne, G. J. Martyna, and M. L. Klein, J. Chem. Phys. 99, 2796 (1993).

${ }^{6}$ J. Barker, J. Chem. Phys. 70, 2914 (1979).

${ }^{7}$ M. F. Herman, E. J. Bruskin, and B. J. Berne, J. Chem. Phys. 76, 5150 (1982).

${ }^{8}$ M. Parrinello and A. Rahman, J. Chem. Phys. 80, 860 (1984).

${ }^{9}$ J. Cao and B. J. Berne, J. Chem. Phys. 91, 6359 (1989).

${ }^{10}$ W. Janke and T. Sauer, J. Chem. Phys. 107, 5821 (1997).

${ }^{11}$ M. Eleftheriou, J. D. Doll, E. Curotto, and D. L. Freeman, J. Chem. Phys. 110, 6657 (1999).

${ }^{12}$ K. R. Glaesemann and L. E. Fried, J. Chem. Phys. 116, 5951 (2002).

${ }^{13}$ K. R. Glaesemann and L. E. Fried, J. Chem. Phys. 117, 3020 (2002).

${ }^{14}$ J. P. Neirotti, D. L. Freeman, and J. D. Doll, J. Chem. Phys. 112, 3990 (2000).

${ }^{15}$ C. Predescu, D. Sabo, J. D. Doll, and D. L. Freeman, J. Chem. Phys. 119, 12119 (2003).

${ }^{16}$ W. Shinoda and M. Shiga, Phys. Rev. E 71, 041204 (2005).

${ }^{17}$ M. Suzuki, Phys. Lett. A 201, 425 (1995).

${ }^{18}$ S. A. Chin, Phys. Lett. A 226, 344 (1997)

${ }^{19}$ M. Takahashi and M. Imada, J. Phys. Soc. Jpn. 53, 3765 (1984).

${ }^{20}$ X.-P. Li and J. Q. Broughton, J. Chem. Phys. 86, 5094 (1987).

${ }^{21}$ N. Makri and W. H. Miller, J. Chem. Phys. 90, 904 (1989).

${ }^{22}$ M. Mella, G. Morosi, and D. Bressanini, Phys. Rev. E 61, 2050 (2000).

${ }^{23}$ S. Jang, S. Jang, and G. A. Voth, J. Chem. Phys. 115, 7832 (2001).

${ }^{24}$ S. A. Chin and C. R. Chen, J. Chem. Phys. 114, 7338 (2001)
${ }^{25}$ R. O. Weht, J. Kohanoff, D. A. Estrin, and C. Chakravarty, J. Chem. Phys. 108, 8848 (1998).

${ }^{26}$ S. D. Bond, B. B. Laird, and B. J. Leimkuhler, J. Chem. Phys. 185, 472 (2003).

${ }^{27}$ F. R. Krajewski and M. H. Müser, Phys. Rev. B 65, 174304 (2002).

${ }^{28}$ S. Chiesa, M. Mella, G. Morosi, and D. Bressanini, J. Chem. Phys. 119, 5601 (2003)

${ }^{29}$ L. Brualla, K. Sakkos, J. Boronat, and J. Casulleras, J. Chem. Phys. 121, 636 (2004).

${ }^{30}$ C. Predescu, Phys. Rev. E 69, 056701 (2004).

${ }^{31}$ S. A. Chin, Phys. Rev. E 69, 046118 (2004).

${ }^{32}$ S. A. Chin, Phys. Rev. E 71, 016703 (2005).

${ }^{33}$ J. Cao and B. J. Berne, J. Chem. Phys. 97, 2382 (1992).

${ }^{34}$ L. M. Sese and R. Ledesma, J. Chem. Phys. 102, 3776 (1995).

${ }^{35}$ A. Bogojevic, A. Balaz, and A. Belic, Phys. Rev. Lett. 94, 180403 (2005).

${ }^{36}$ C. Chakravarty, M. C. Gordillo, and D. M. Ceperley, J. Chem. Phys. 109, 2123 (1998)

${ }^{37}$ A. Nakayama and N. Makri, J. Chem. Phys. 119, 8592 (2003); Chem. Phys. 304, 147 (2004); Proc. Natl. Acad. Sci. U.S.A. 102, 4230 (2005).

${ }^{38}$ For $\mathbf{x}^{*}=\mathbf{x}_{P}$ the $P$ th term in the square bracket in Eq. (6) vanishes, so that integration by parts of the remaining $P-1$ terms gives $g=1$. The result for $\mathbf{x}^{*}=\mathbf{x}_{c}$ can be obtained via cyclic averaging of a similar equation with $\mathbf{x}^{*}=\mathbf{x}_{t}, t=1, \ldots, P$.

${ }^{39}$ This can be shown by differentiating the relation $K\left(\left\{\mathbf{x}^{*}+\lambda\left(\mathbf{x}_{s}-\mathbf{x}^{*}\right)\right\}\right)$ $=\lambda^{2} K\left(\left\{\mathbf{x}_{s}\right\}\right)$ with respect to $\lambda$ and setting $\lambda$ to 1 , where $K\left(\left\{\mathbf{x}_{s}\right\}\right)$ is the kinetic action in Eq. (3).

${ }^{40}$ In this paper we consider only smooth potentials that have continuous first- and second-order derivatives.

${ }^{41}$ J. D. Doll and D. L. Freeman, J. Chem. Phys. 111, 7685 (1999).

${ }^{42}$ C. Predescu, D. Sabo, J. D. Doll, and D. L. Freeman, J. Chem. Phys. 119, 10475 (2003).

${ }^{43}$ In the classical calculation we used the replica-exchange Monte Carlo to avoid possible nonergodicity problem in the solidlike phase at low temperature. The replica temperatures were distributed over $[6,30] \mathrm{K}$ with an even spacing of $1 \mathrm{~K}$, which ensured $>40 \%$ acceptance ratio for the exchange move.

${ }^{44}$ C. Chakravarty, J. Chem. Phys. 102, 956 (1995).

${ }^{45}$ F. Calvo, J. P. K. Doye, and D. J. Wales, J. Chem. Phys. 114, 7312 (2001).

${ }^{46}$ V. Buch, J. Chem. Phys. 117, 4738 (2002).

${ }^{47}$ P. A. Frantsuzov and V. A. Mandelshtam, J. Chem. Phys. 121, 9247 (2004).

${ }^{48}$ C. Predescu, P. A. Frantsuzov, and V. A. Mandelshtam, J. Chem. Phys. 122, 154305 (2005).

${ }^{49}$ E. Marinari and G. Parisi, Europhys. Lett. 19, 451 (1992).

${ }^{50}$ K. Hukushima and K. Nemoto, J. Phys. Soc. Jpn. 65, 1604 (1996).

${ }^{51}$ U. H. E. Hansmann, Chem. Phys. Lett. 281, 140 (1997).

${ }^{52}$ W. H. Miller, S. D. Schwartz, and J. W. Tromp, J. Chem. Phys. 79, 4889 (1983)

${ }^{53}$ T. Yamamoto and W. H. Miller, J. Chem. Phys. 120, 3086 (2004).

${ }^{54}$ Y. Zhao, T. Yamamoto, and W. H. Miller, J. Chem. Phys. 120, 3100 (2004).

${ }^{55}$ T. Yamamoto and W. H. Miller, J. Chem. Phys. 122, 044106 (2005).

${ }^{56}$ C. Predescu, Phys. Rev. E 70, 066705 (2004).

${ }^{57}$ E. Rabani and D. R. Reichman, J. Phys. Chem. B 105, 6550 (2001).

${ }^{58}$ A. Sarsa, K. E. Schmidt, and W. R. Magro, J. Chem. Phys. 113, 1366 (2000).

${ }^{59}$ J. E. Cuervo, P.-N. Roy, and M. Boninsegni, J. Chem. Phys. 122, 114504 (2005). 